\title{
CHEMICAL INHOMOGENEITY IN RED GIANT BRANCH STARS AND RR LYRAE VARIABLES IN NGC 1851: TWO SUBPOPULATIONS IN RED GIANT BRANCH*
}

\author{
Jae-Woo LeE ${ }^{1,5}$, Jina Lee ${ }^{1,5}$, Young-Woon Kang ${ }^{1}$, Young-Wook LeE ${ }^{2}$, Sang-Il Han ${ }^{2}$, Seok-Joo Joo ${ }^{2}$, Soo-Chang Rey ${ }^{3}$, \\ AND DAVID YONG ${ }^{4}$ \\ ${ }^{1}$ Department of Astronomy and Space Science, Astrophysical Research Center for the Structure and Evolution of the Cosmos, Sejong University, 98 Gunja-Dong, \\ Gwangjin-Gu, Seoul 143-747, Korea; jaewoolee@sejong.ac.kr, kangyw@sejong.ac.kr \\ ${ }^{2}$ Center for Space Astrophysics and Department of Astronomy, Yonsei University, Shinchon 134, Seoul 120-749, Korea; ywlee@csa.yonsei.ac.kr \\ ${ }^{3}$ Department of Astronomy and Space Science, Chungnam National University, Daejeon 305-764, Korea; screy@cnu.ac.kr \\ ${ }^{4}$ Research School of Astronomy and Astrophysics, Australian National University, Weston, ACT 2611, Australia; yong@mso.anu.edu.au \\ Received 2008 December 29; accepted 2009 February 20; published 2009 March 23
}

\begin{abstract}
We investigate the red giant branch (RGB) subpopulations of NGC 1851 using Ca uvby photometry. Our colormagnitude diagrams show that the RGB stars have two subpopulations and the RGB stars in NGC 1851 appear to have distinct elemental abundance patterns with the $[\mathrm{Ca} / \mathrm{H}]$ abundance. We discuss that the elemental abundance patterns can be explained by the contributions from the asymptotic giant stars, confirming the previous studies by others. The RR Lyrae variables in NGC 1851 appear to have a large metallicity spread and, perhaps, a bimodal metallicity distribution. Our period shift analysis of the RR Lyrae variables shows that the helium enhancement appears to be insignificant in the NGC 1851 RR Lyrae population. However, the helium enrichment scenario in the blue or the red parts of horizontal branch (HB) cannot be completely ruled out. Our results show that about $18 \%$ of the bright RGB stars have enhanced CNO abundances, sharply in contrast to previous estimates by others ( $\approx 38 \%)$, making it difficult to explain the double subgiant, RGB sequences, and the bimodal HB distribution by their number ratios.
\end{abstract}

Key words: globular clusters: individual (NGC 1851) - Hertzsprung-Russell diagram - stars: abundances - stars: evolution

\section{INTRODUCTION}

During the last few years, it has become increasingly clear that some Galactic globular clusters (GC) contain multiple stellar populations. The most vivid examples are $\omega$ Cen (Lee et al. 1999; Bedin et al. 2004), NGC 2808 (Piotto et al. 2007; D’Antona et al. 2005), NGC 6388 (Piotto 2008), and NGC 1851 (Milone et al. 2008).

Recent studies indicate that NGC 1851 is one of the most intriguing GCs in many aspects. First, NGC 1851 shows a double subgiant branch (SGB) with no evidence of main-sequence (MS) splitting, which lead Milone et al. (2008) to conclude that NGC 1851 may have experienced at least two star formation histories within a time span of $\approx 1 \mathrm{Gyr}$, depending on the detailed chemical compositions of the subpopulations. In contrast to $\omega$ Cen or NGC 2808, the helium enhancement does not appear to occur significantly and the bimodal horizontal branch (HB) morphology of NGC 1851 can be explained without invoking large helium enhancement (Cassisi et al. 2008). Second, NGC 1851 appears to have bimodal chemical compositions. Hesser et al. (1982) showed that some red giant branch (RGB) stars in NGC 1851 have extremely strong CN band strengths presumably due to extremely strong $\mathrm{C}$ and/or $\mathrm{N}$ abundances, which was confirmed by Yong et al. (2009) who found enhanced N abundances in the CN-strong RGB stars. More recently, Yong \& Grundahl (2008) and Yong et al. (2009) studied RGB stars in NGC 1851 employing high-resolution spectroscopy and their results show that NGC 1851 exhibits large star-to-star abundance variations including the CNO abundance. The $s$-process

\footnotetext{
* Based on observations made with the CTIO $1 \mathrm{~m}$ telescope, which is operated by the SMARTS consortium.

5 Visiting Astronomer, Cerro Tololo Inter-American Observatory (CTIO), National Optical Astronomy Observatories, operated by the Association of Universities for Research in Astronomy, Inc., under cooperative agreement with the National Science Foundation.
}

elemental abundances, [Zr/Fe] and [La/Fe], in NGC 1851 appear to be bimodal and the $\mathrm{Na}, \mathrm{Al}, \mathrm{Zr}$, and $\mathrm{La}$ abundances are correlated with the $\mathrm{CNO}$ abundance. In particular, Yong et al. (2009) concluded that NGC 1851 is the first cluster providing strong support for the asymptotic giant branch (AGB) pollution scenario for the star-to-star light elemental abundance variations. Third, the interstellar reddening toward NGC 1851, $E(B-V) \approx 0.02 \mathrm{mag}$ (Harris 1996), is very small compared to other GCs showing multiple stellar populations such that differential reddening may be negligible, making the interpretation of the observational data relatively straightforward.

In this Letter, we investigate the multiple stellar populations in RGB and the metallicity spread of the RR Lyrae variables in NGC 1851 using Ca uvby photometry.

\section{OBSERVATIONS AND DATA REDUCTION}

Ca uvby observations were made during 2008 February $12-15$ and 2008 August 5-11 using the CTIO $1.0 \mathrm{~m}$ telescope as a part of Sejong/ARCSEC Ca uvby survey aimed at obtaining Ca $u v b y$ photometry for $\geqslant 50 \mathrm{GCs}$ and selected fields in Baade's windows $\left(\geqslant 3 \mathrm{deg}^{2}\right)$ led by the first author. The telescope was equipped with an STA $4 \mathrm{k} \times 4 \mathrm{k}$ CCD camera, providing a plate scale of $0.289 \operatorname{arcsec}_{\text {pixel }}^{-1}$ and a field of view of $20 \times 20$ arcmin. The total exposure times were 19,100, 12,300, 7400, 7100 , and $3810 \mathrm{~s}$ for Ca, Strömgren $u, v, b$, and $y$, respectively. We also obtained bias and twilight or dawn sky flat images to calibrate science exposures. Five nights were photometric and we observed about 60 standards from Twarog \& AnthonyTwarog (1995) and Anthony-Twarog \& Twarog (1998). The photometry of the cluster and standard frames were analyzed using DAOPHOTII, ALLFRAME, and DAOGROW (Stetson $1987,1990,1994)$ following the method described in Lee \& Carney (1999a). 

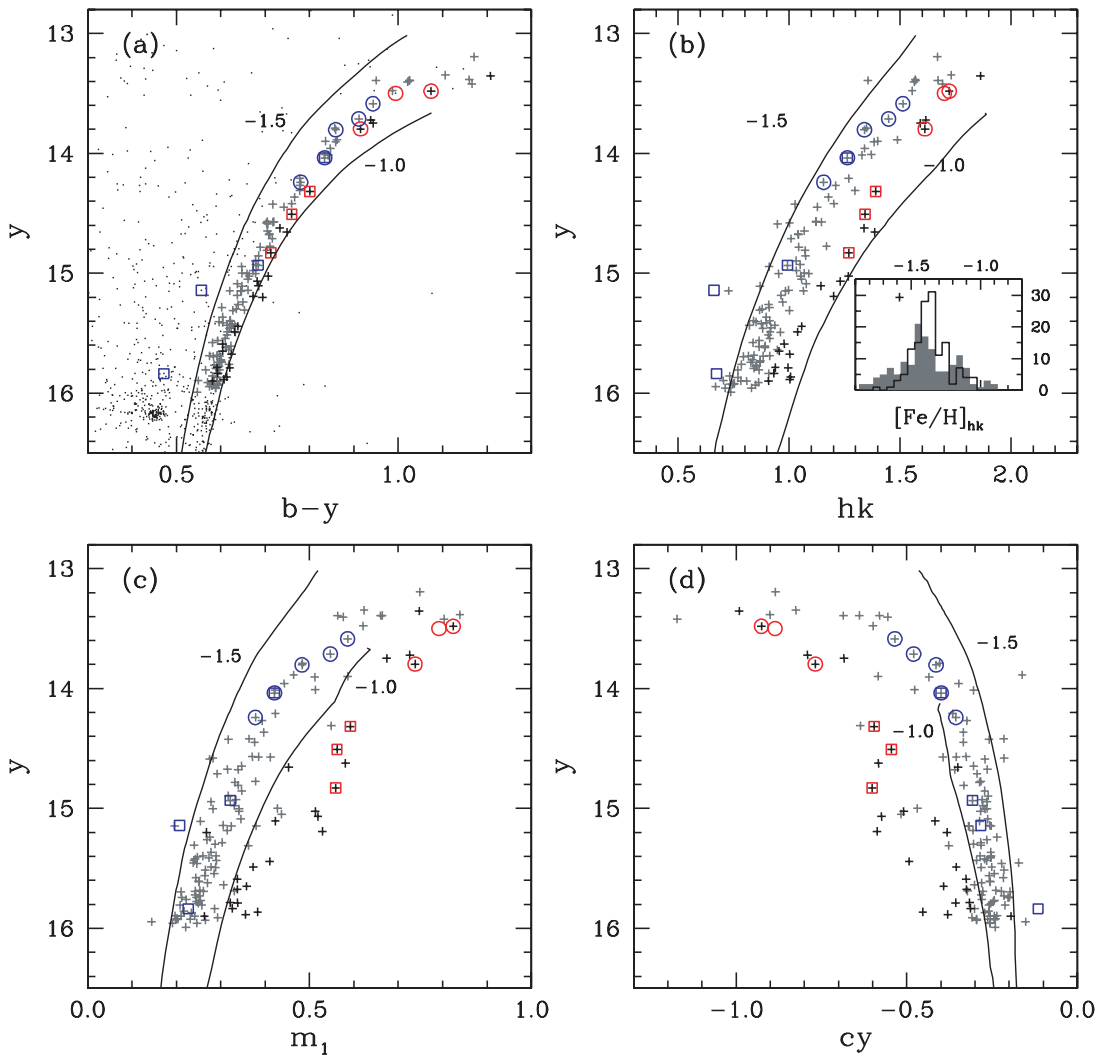

Figure 1. CMDs for RGB stars in NGC 1851. The RGB- $n$ stars are denoted by the light gray plus signs and the RGB- $s$ stars are denoted by the black plus signs. RGB stars studied by Hesser et al. (1982) are denoted by the open squares and those studied by Yong \& Grundahl (2008) by open circles. The red color indicates the RGB- $s$ population, while the blue color indicates the RGB- $n$ population. The metallicity grids for $[\mathrm{Fe} / \mathrm{H}]=-1.0$ and -1.5 with $[\alpha / \mathrm{Fe}]=0.4 \mathrm{dex}$ are also shown. In the inset of (b), the metallicity distributions of the RGB stars (black and gray plus signs) using the $h k$ vs. $y$ (the solid line) and the $h k$ vs. $b-y$ (the shaded area) relations are shown.

\section{RESULTS}

\subsection{The Double RGB Sequence}

We show the color-magnitude diagrams (CMDs) of the bright RGB stars $(V \leqslant 16 \mathrm{mag})$ in Figure 1 . In the figure, we do not attempt to remove the off-cluster field stars in our sample since both the proper motion and the radial velocity measurements are not available. Being a high galactic latitude system $\left(b=-35^{\circ}\right)$, the contribution from the field star contamination is expected to be small but our RGB selection criterion is not based on the proper motion study or the radial velocity measurements. Therefore, our statistics could be slightly incorrect. Future studies on the proper motion and the radial velocity measurements would be desirable.

In Figure 1(a), black and gray plus signs represent RGB stars between 0.5 and 5'.0 from the cluster's center ${ }^{6}$ (141 stars) and black dots represent all stars measured in our images. We also show RGB stars studied by Hesser et al. (1982), Yong \& Grundahl (2008), and $Y^{2}$ isochrones for $[\mathrm{Fe} / \mathrm{H}]=-1.5,-1.0$ with $10 \mathrm{Gyr}$ (Yi et al. 2003). Since the $Y^{2}$ isochrones do not provide colors in the Ca uvby system, we calculate $(b-y), h k$ [ $=(\mathrm{Ca}-b)-(b-y)], m_{1}$, and $c y\left[=c_{1}-(b-y)\right.$; Yong et al. 2008] using the color-temperature relations given by Castelli ${ }^{7}$ and we use $E(B-V)=0.02$ mag and the distance modulus of $15.47 \mathrm{mag}$ for NGC 1851 (Harris 1996). As noted by others, the RGB sequence of NGC 1851 is broader than those of other GCs

\footnotetext{
6 Note that the tidal radius of NGC 1851 is 11 '7 (Harris 1996).

7 http://wwwuser.oat.ts.astro.it/castelli/colors/uvbyca.html.
}

in $B-V, b-y$, and $V-I$ CMDs, but it is difficult to see distinct multiple RGB populations in our $(y, b-y)$ CMD.

Figure 1(b) shows a plot of $y$ versus $h k$. The Ca filter system measures $\mathrm{Ca}$ II $\mathrm{H}$ and $\mathrm{K}$ lines and the $h k$ index is known to be about three times more sensitive to metallicity than the $m_{1}$ index is for stars more metal-poor than the Sun (Anthony-Twarog et al. 1991). However, the $h k$ index is vulnerable to the influence of $\mathrm{H} \epsilon$ for hotter stars, interstellar Ca II $\mathrm{H}$ and $\mathrm{K}$ absorption lines, and chromospheric activity. In the figure, the RGB stars in NGC 1851 appear to split into the two populations ${ }^{8}$ at $[\mathrm{Fe} / \mathrm{H}] \approx-1.25$ $\operatorname{dex}($ equivalent to $[\mathrm{Ca} / \mathrm{H}] \approx-0.85 \mathrm{dex}$, where $[\alpha / \mathrm{Fe}]=+0.4$ dex); one with the normal $h k$ index (115 stars: $\approx 82 \%$ of the total RGB stars, RGB- $n$ hereafter), whose metallicities derived from the $h k$ index are consistent with those from high-resolution spectroscopy, and the other with the strong $h k$ index (26 stars: $\approx 18 \%$ of the total RGB stars, RGB-s hereafter). At first glance, it was thought that the RGB-s stars may belong to the offcluster population with larger metal contents. However, at least five of the RGB-s stars ${ }^{9}$ are radial velocity members of NGC 1851 (Hesser et al. 1982; Yong \& Grundahl 2008). In particular, we note that the three calcium-enhanced stars, which will be discussed below, studied by Yong \& Grundahl (2008) and the three $\mathrm{CN}$-strong stars with enhanced $\mathrm{Sr}$ II and $\mathrm{Ba}$ II abundances from Hesser et al. (1982) lie on the RGB- $s$ sequence. In the inset of Figure 1(b), we show the metallicity distributions of

\footnotetext{
8 Calamida et al. (2007) first suggested that NGC 1851 appears to have double RGB sequences (see their Figure 9).

9 The star 395 of Yong \& Grundahl (2008) is located at $>5^{\prime}$ from the cluster center.
} 


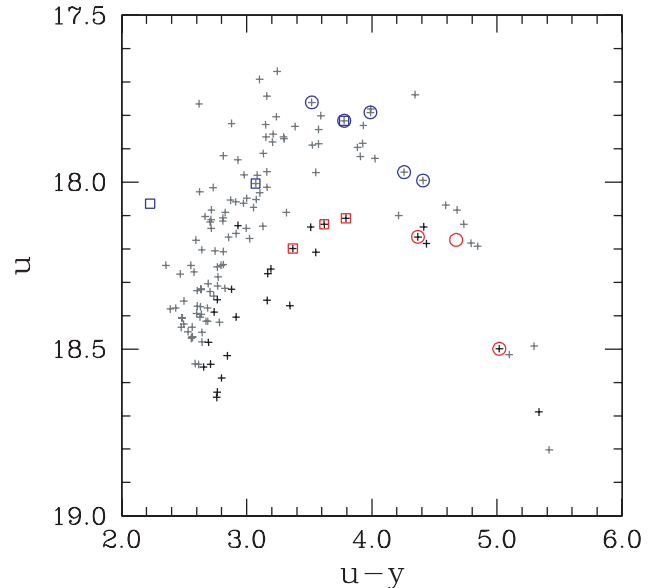

Figure 2. $u$ vs. $u-y$ CMD of our sample RGB stars in NGC 1851. Notations are the same as in Figure 1.

our RGB stars using the $h k$ versus $y$ (the solid line) and the $h k$ versus $b-y$ (the shaded area) relations. These two metallicity distributions do not match perfectly each other, but the presence of the RGB-s population can be seen in both distributions.

The double RGB sequence persists on the $\left(y, m_{1}\right)$ and the $(y, c y)$ CMDs as shown in Figures 1(c) and (d) but a double RGB is not seen in the $(y, b-y)$ CMD. It should be noted that, however, the double RGB sequence in Figures 1(c) and (d) may be expected from the results of Hesser et al. (1982). They showed that the CN band strengths in some NGC 1851 RGB stars was comparable to that seen in a very peculiar RGB star in $\omega$ Cen. The Strömgren $u, v$ bandpasses include the CN bands and, as a consequence, the $m_{1}, c_{1}$, and $c y$ indices are also affected by the $\mathrm{CN}$ band strengths. Comparisons of Figure 1(b) with Figure 1(c) or (d) may suggest that the $\mathrm{Ca}$ II $\mathrm{H}$ and $\mathrm{K}$ line strengths trace the $\mathrm{CN}$ band strengths in NGC 1851 RGB stars. The correlation between the calcium abundances and the $\mathrm{CN}$ band strengths is also supported by Ivans et al. (1999), who found the similar correlation in the GC M4.

Perhaps, the double RGB sequence in NGC 1851 can be more clearly seen in Figure 2. The $u-y$ color is more sensitive to temperature than the $b-y$ color is (Calamida et al. 2007), and the Strömgren $u$ bandpass is sensitive to the $\mathrm{NH}$ and the $\mathrm{CN}$ band strengths. Note that the $\mathrm{CN}$-strong RGB stars are Narich, O-poor, and the $\mathrm{CN}$-weak RGB stars are Na-poor, O-rich in NGC 1851 (see below). Although the number ratios between the $\mathrm{CN}$-strong and the $\mathrm{CN}$-weak RGB stars are different, the NGC 1851 RGB stars behave the same as the double RGB sequence in M4 (Marino et al. 2008).

In Figure 3, we show elemental abundances from highresolution spectroscopy (Yong \& Grundahl 2008; Yong et al. 2009; D. Yong \& F. Grundahl 2009, in preparation) as functions of $[\mathrm{Ca} / \mathrm{H}]$ for eight RGB stars in NGC 1851 . We adopt $[\mathrm{Ca} /$ $\mathrm{H}]$ as a reference because our $h k$ index traces the $[\mathrm{Ca} / \mathrm{H}]$ abundance, not the $[\mathrm{Fe} / \mathrm{H}]$ abundance. Note that the star-to-star $[\mathrm{Ca} / \mathrm{H}]$ variations in NGC $1851 \mathrm{RGB}$ stars from high-resolution spectroscopy can be as large as $\Delta[\mathrm{Ca} / \mathrm{H}] \approx 0.3 \mathrm{dex}$, consistent with our photometric estimates shown in Figure 1(b). Within the limited sample size, the RGB stars in NGC 1851 appear to have two distinctive elemental abundance patterns with the boundary at $[\mathrm{Ca} / \mathrm{H}] \approx-0.80 \mathrm{dex}$. As Yong \& Grundahl (2008) discussed, the $s$-process element $\mathrm{La}$ (and perhaps $\mathrm{Zr}$ ) clearly shows the bimodal distribution in the figure. The light elements $\mathrm{N}, \mathrm{O}, \mathrm{Na}$, and $\mathrm{Al}$ also show the bimodal distributions against

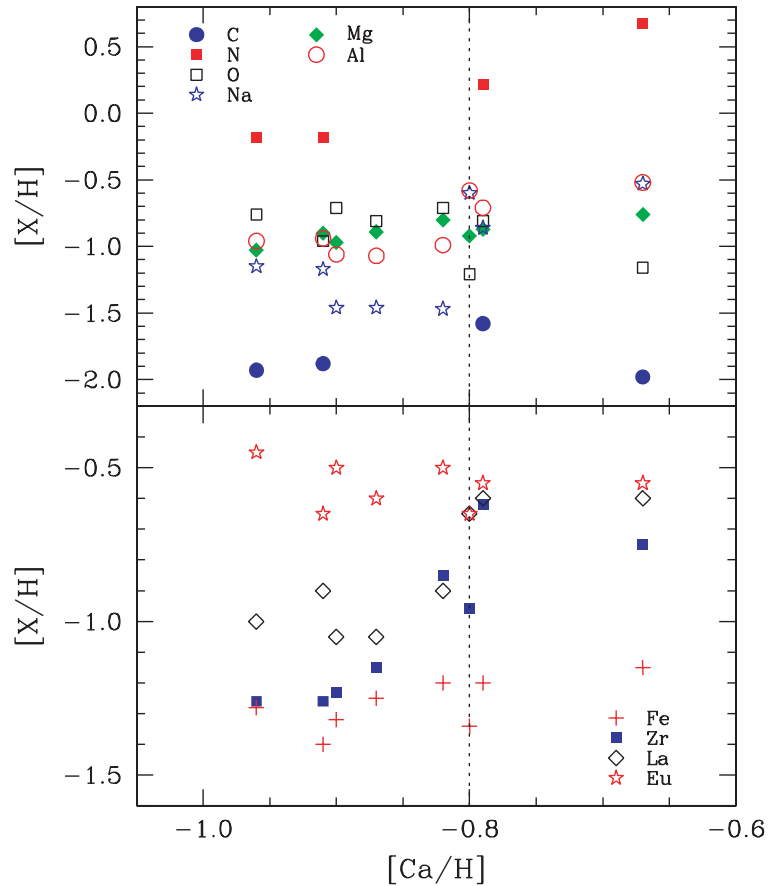

Figure 3. Elemental abundances against $[\mathrm{Ca} / \mathrm{H}]$ of eight RGB stars in $\mathrm{NGC}$ 1851 (Yong \& Grundahl 2008; Yong et al. 2009; D. Yong \& F. Grundahl, in preparation). The RGB stars in NGC 1851 appear to have two distinct elemental abundance patterns with the boundary at $[\mathrm{Ca} / \mathrm{H}]=-0.80$ dex.

$[\mathrm{Ca} / \mathrm{H}]$, while $\mathrm{Mg}, \mathrm{Fe}$ (perhaps $\mathrm{Zr}$ as noted by the referee) appear to be linearly correlated with $[\mathrm{Ca} / \mathrm{H}]$. On the other hand, the $r$-process element Eu does appear to vary against $[\mathrm{Ca} / \mathrm{H}]$.

Yong et al. (2008) showed that the $c y$ index is correlated with the nitrogen abundance in NGC 6752 and that all clusters show a large spread in the $c y$ index at a given luminosity presumably driven by large nitrogen variations at all luminosities. If this correlation between the $c y$ index and the nitrogen abundance is still valid for NGC 1851, then the three CN-strong, $c y$-weak RGB stars from Hesser et al. (1982) should be nitrogen-poor compared to the CN-normal RGB stars in NGC 1851. But the opposite is found, that is, the $\mathrm{CN}$-strong stars are nitrogenrich (Yong et al. 2009). The typical RGB stars in GCs show an anticorrelation between the $\mathrm{CN}$ band and the $\mathrm{CH}$ band strengths and a correlation between the $\mathrm{CN}$ band and the $\mathrm{NH}$ band strengths, indicating that the nitrogen controls the $\mathrm{CN}$ band strength (see, for example, Briley \& Smith 1993). It is thought that the $\lambda 4215 \mathrm{CN}$ band strongly affects the Strömgren $v$ bandpass and the correlation between the $c y$ index and the nitrogen abundance found in NGC 6752 does not work for NGC 1851.

\subsection{The Metallicity Spread and the Helium Contents in the RR Lyrae Stars}

The $h k$ index can also be used as a metallicity indicator for RR $a b$-type variables in place of the $\Delta S$ method. The advantage of using the $h k$ index is that the metallicities derived from the $h k$ index are independent of the pulsational cycle of the variables (Baird 1996). In Figure 4(a), we show a metallicity distribution of 21 RRab-type variables in NGC 1851 (Walker 1998) using the $h k$ versus $[\mathrm{Fe} / \mathrm{H}]$ relations given by Rey et al. (2000). As in the RGB sequence, RR Lyrae variables can be divided into two subgroups at $[\mathrm{Fe} / \mathrm{H}]=-1.2 \mathrm{dex}$ : the $h k$-normal $(12$ variables: $57 \%$ of RR Lyrae) and the $h k$-strong (nine variables: 

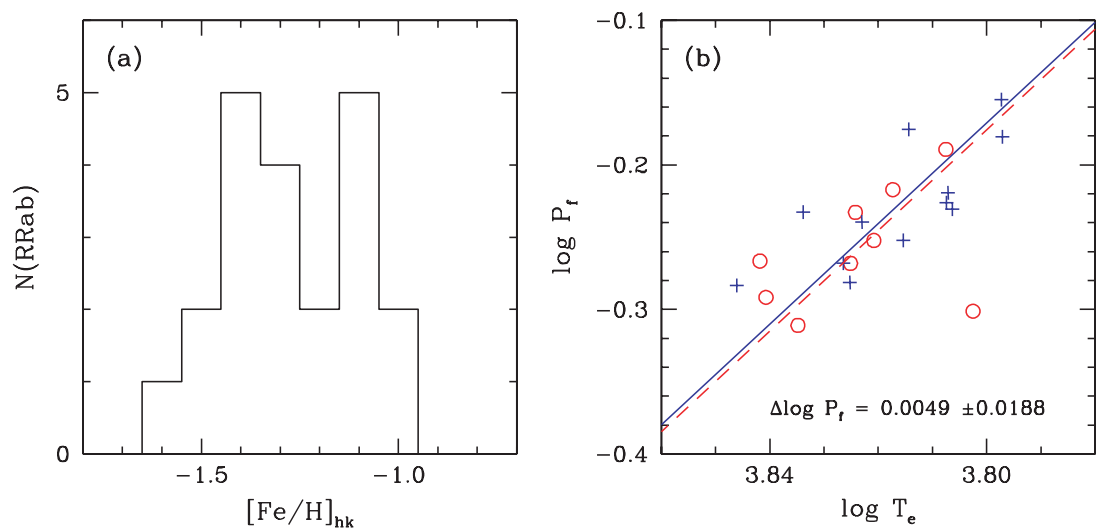

Figure 4. (a) Metallicity distribution of $21 \mathrm{RR} a b$-type variables in NGC 1851 using the $h k$ index. (b) Plot of $\log T_{\mathrm{e}}$ vs. $\log P_{\mathrm{f}}$ for RRab-type variables in NGC 1851 . The blue plus signs and the red circles represent the $h k$-normal (12 stars) and the $h k$-strong (nine stars) RR Lyrae variables, respectively. The blue solid line and the red dashed line represent linear fits to the $h k$-normal and the $h k$-strong RR Lyrae variables, respectively.

$43 \%$ of RR Lyrae) populations. The small sample size is an unavoidable problem as noted by the referee and the rms scatter related with the $[\mathrm{Fe} / \mathrm{H}]_{h k}$ calibration is rather large, 0.12 dex (Rey et al. 2000). Therefore, our number statistics may not be considered to be correct and the metallicity distribution shown in Figure 4 may tell us nothing more than a metallicity spread in the NGC 1851 RR Lyrae variables. It should be emphasized that, however, whether a bimodal RR Lyrae metallicity distribution or not, the metallicity spread in NGC 1851 RR Lyrae variables, $\Delta[\mathrm{Fe} / \mathrm{H}]_{h k} \approx 0.5 \mathrm{dex}$, in addition to the potential age spread inferred from the double SGB sequence appears to be enough to explain the bimodal HB distribution seen in NGC 1851.

The enhanced helium abundances, such as widely accepted in $\omega$ Cen and NGC 2808, can also produce the bimodal HB distribution. The idea is that the second generation stars in such clusters have enhanced helium and metal abundances and they are in the blue part of the HB morphology. The trouble with this idea is that direct measurement of the helium abundance for GC stars is not feasible. The period shift analysis of RR Lyrae variables may provide an indirect means of estimating the helium abundances of the variables, in the sense that higher helium abundance makes RR Lyrae variables brighter and, as a consequence, higher helium abundance makes their pulsational period longer.

In Figure 4(b), we show a plot of $\log T_{\mathrm{e}}$ versus $\log P_{\mathrm{f}}$ for RR $a b$-type variables, where $T_{\mathrm{e}}$ is the equilibrium temperature of variables (see Lee \& Carney 1999b and references therein) and $P_{\mathrm{f}}$ is the period of RR $a b$-type variables, and we adopt those from Walker (1998). The linear fits to the data are also shown and we find $\Delta \log P_{\mathrm{f}}=0.0049 \pm 0.0188$, in the sense that the $h k$-normal RR Lyrae population has slightly longer mean period. To reproduce the observed bimodal HB distribution of NGC 1851 with the helium abundance variations, a large amount of helium enhancement, $\Delta Y \geqslant 0.05$, is required. This large amount of helium enhancement strongly affects the period shift, $\Delta \log P_{\mathrm{f}} \geqslant+0.04$ (see, for example, Figures 10 and 11 in Lee et al. 1994), and this expected period shift appears to be very large compared to our result. Therefore, our result may suggest that the helium abundances of the $h k$-strong and the $h k$-normal RR Lyrae populations are very similar. Note that our period shift analysis does not provide any information on the inferred RHB or BHB helium abundances. Although no evidence of MS splitting was found (Milone et al. 2008), the helium enhancement in some part of the BHB or the RHB stars cannot be completely ruled out.

\section{DISCUSSIONS}

In this Letter, we showed that the RGB and the RR Lyrae variables in NGC 1851 also have the double populations. Qualitatively, the bimodal elemental abundance patterns except for the calcium abundance appear to be consistent with the AGB contributions to the second generation of the stars in NGC 1851 (see Yong \& Grundahl 2008; Yong et al. 2009, for detailed discussions). However, the star-to-star calcium (and perhaps $\mathrm{Fe}$ ) abundance variation cannot be easily explained by the (single star) AGB contributions. Although small, $\Delta[\mathrm{Ca} / \mathrm{H}] \approx 0.3 \mathrm{dex}$ appears to exist in NGC 1851 and, furthermore, other elemental abundances appear to be correlated or anticorrelated with $[\mathrm{Ca} /$ $\mathrm{H}$ ] as shown in Figure 3, suggesting that the calcium abundance variation may not be primordial. The majority of the calcium is known to be synthesized in the Type II supernovae, which does not appear to be the case since the RGB stars in NGC 1851 do not show star-to-star $[\mathrm{Eu} / \mathrm{H}]$ variations, while parts of calcium isotopes can also be synthesized via the $s$-process. But the $s$-process contributions are thought to be negligibly small (Clayton 2003). The observed light elemental abundance anomalies in GCs can be created by the ejected material from the first generation of fast rotating massive stars (Decressin et al. 2007), but it is not clear whether slow winds from such stars can enhance the calcium abundance of the second generation stars.

The RR Lyrae variables in NGC 1851 also show the star-tostar calcium abundance variation as can be seen in the RGB stars and further there appears no helium enhancement in the NGC 1851 RR Lyrae variables.

Qualitatively, assuming two star formation histories, the double SGB sequence (Milone et al. 2008) and the bimodal CNO abundance (Cassisi et al. 2008; Yong et al. 2009) appears to nicely explain the bimodal HB distribution of NGC 1851 . However, the quantitative analysis of number ratios of the multiple populations between SGB, RGB, and HB may tell a different scenario. Based on our number statistics, it is not clear whether the fSGB, which presumably has enhanced CNO abundances with younger or coeval age as suggested by others (Cassisi et al. 2008; Salaris et al. 2008; Yong \& Grundahl 2008), have evolved to the BHB population via the RGB-s population. The number of the RGB-s population (18\% of the total RGB stars) appears to be too small to being the progeny of the fSGB (45\% of the total SGB stars, respectively) or the progenitor of the entire BHB population $(37 \% \pm 9 \%$ of the total RHB + BHB stars; Milone et al. 2008) in NGC 1851. We emphasize that the frequently cited value for the fraction of 
the $C N$-strong $R G B$ (38\%, three out of eight; Hesser et al. 1982) by others, in an attempt to explain the bimodal HB distribution in conjunction with the fSGB/bSGB and the $\mathrm{CN}$-strong/CNweak RGB populations, may not be correct. This value should be referred as "the fraction of the $\mathrm{CN}$-strong bright RGB + AGB." In fact, Hesser et al. (1982) measured CN strengths for 16 or 18 RGB stars, depending on the evolutionary status of the stars R6 and S32 (potentially being AGBs as noted by Hesser et al. 1982). Therefore, the correct fraction of the CN-strong RGB stars by Hesser et al. (1982) should be either $17 \%$ (three out of 18 ) or $19 \%$ (three out of 16), very consistent with our result $(18 \%)$.

In contrast to previous studies, we find it very difficult to explain the number ratio between the RGB- $n$ and the RGB$s$ populations in relation to the double SGB sequence and the bimodal HB distribution, unless (1) each subpopulation has very different luminosity functions (LFs), or (2) either the fSGB or the bSGB population splits on the RGB sequence in our CMDs as can be seen in M4 (Marino et al. 2008).

Since the physical environment at the formation epoch of the second generation of stars could have been different from that of the first generation, it may be natural to expect that the LF for the first generation is different from that for the second generation. This can explain the differences in number statistics of subpopulations between the double SGB (bSGB:fSGB $\approx$ 65:45) and the double RGB sequences (RGB- $n:$ RGB- $s \approx$ $4: 1$ ). However, this approach still poses a difficult problem in explaining the differences in the number ratios between the RGB (RGB- $n:$ RGB- $s \approx 4: 1)$ and the HB (RHB:BHB $\approx 3: 2$ ).

On the other hand, the recent study of the GC M4 (Marino et al. 2008) may strongly support our idea that either the fSGB or the bSGB splits into two RGB sequences. As Marino et al. (2008) noted, no evidence of an SGB and an MS splits can be found in M4 from very accurate photometry based on ACS/HST. However, the RGB stars in M4 split into two separate sequences in the $(U, U-B) \mathrm{CMD}$ and, furthermore, each RGB subpopulation in M4 has distinctive elemental abundance patterns as can be seen in NGC 1851. Due to the accuracy of our photometry at the SGB level, we are not able to identify which SGB splits into two sequences. However, it can be verified by studying the fraction of the CN-strong SGB stars in the bSGB/ fSGB populations and the spectroscopic study of the SGB stars in NGC 1851 is desperately needed in the future.
We thank an anonymous referee for helpful comments and a careful review of the Letter. Support for this work was provided by the Korea Science and Engineering Foundation (KOSEF) to the Astrophysical Research Center for the Structure and Evolution of the Cosmos (ARCSEC").

\section{REFERENCES}

Anthony-Twarog, B. J., Laird, J. N., Payne, D., \& Twarog, B. A. 1991, AJ, 101, 1902

Anthony-Twarog, B. J., \& Twarog, B. A. 1998, AJ, 116, 1922

Baird, S. R. 1996, AJ, 112, 2132

Bedin, L. R., Piotto, G., Anderson, J., Cassisi, S., King, I. R., Momany, Y., \& Carraro, G. 2004, ApJ, 605, L125

Briley, M. M., \& Smith, G. H. 1993, PASP, 105, 1260

Calamida, A., et al. 2007, ApJ, 670, 400

Cassisi, S., Salaris, M., Pietrinferni, A., Piotto, G., Milone, A. P., Bedin, L. R., \& Anderson, J. 2008, ApJ, 672, L115

Clayton, D. 2003, Handbook of Isotopes in the Cosmos: Hydrogen to Gallium (Cambridge: Cambridge Univ. Press)

D’Antona, F., Bellazzini, M., Caloi, V., Pecci, F. F., Galleti, S., \& Rood, R. T. 2005, ApJ, 631, 868

Decressin, T., Meynet, G., Charbonnel, C., Prantzos, N., \& Ekström, S. 2007, A\&A, 464, 1029

Harris, W. E. 1996, AJ, 112, 1487

Hesser, J. E., Bell, R. A., Harris, G. L. H., \& Cannon, R. D. 1982, AJ, 87, 1470

Ivans, I., et al. 1999, AJ, 118, 1273

Lee, J.-W., \& Carney, B. W. 1999a, AJ, 117, 2868

Lee, J.-W., \& Carney, B. W. 1999b, AJ, 118, 1373

Lee, Y.-W., Demarque, P., \& Zinn, R. 1994, ApJ, 423, 248

Lee, Y.-W., Joo, J.-M., Sohn, Y.-J., Rey, S.-C., Lee, H.-c., \& Walker, A. R. 1999, Nature, 402, 55

Marino, A. F., et al. 2008, A\&A, 490, 625

Milone, A. P., et al. 2008, ApJ, 673, 241

Piotto, G. 2008, MmSAI, 79, 334

Piotto, G., et al. 2007, ApJ, 661, L53

Rey, S.-C., Lee, Y.-W., Joo, J.-M., Walker, A., \& Baird, S. 2000, AJ, 119, 1824

Salaris, M., Cassisi, S., \& Pietrinferni, A. 2008, ApJ, 678, L25

Stetson, P. B. 1987, PASP, 99, 191

Stetson, P. B. 1990, PASP, 102, 932

Stetson, P. B. 1994, PASP, 106, 250

Twarog, B. A., \& Anthony-Twarog, B. J. 1995, AJ, 109, 2828

Walker, A. R. 1998, AJ, 116, 220

Yi, S. K., Demarque, P., \& Kim, Y.-C. 2003, ApJS, 144, 259

Yong, D., \& Grundahl, F. 2008, ApJ, 672, L29

Yong, D., Grundahl, F., D’Antona, F., Karakas, A. I., Lattanzio, J. C., \& Norris, J. E. 2009, ApJL, in press (arXiv:0902.1773)

Yong, D., Grundahl, F., Johnson, J., \& Asplund, M. 2008, ApJ, 684, 1159 